\title{
Soybean Microbiome Recovery After Disruption is Modulated by the Seed and Not the Soil Microbiome
}

\author{
Itumeleng Moroenyane, ${ }^{1}$ Julien Tremblay, ${ }^{2}$ and Étienne Yergeau ${ }^{1, \dagger}$ \\ ${ }^{1}$ Institut National de la Recherche Scientifique, Centre Armand-Frappier Santé Biotechnologie, 531 Boulevard des Prairies, Laval, Québec, \\ H7V1B7, Canada \\ ${ }^{2}$ Energy, Mining, and Environment, Natural Research Council Canada, 6100 Avenue RoyalMount, Montreal, Quebec, H4P 2R2, Canada
}

Accepted for publication 12 May 2021.

\begin{tabular}{|c|c|}
\hline \multicolumn{2}{|c|}{ ABSTRACT } \\
\hline $\begin{array}{l}\text { Endophytic microbiomes of healthy seed form a symbiotic } \\
\text { relationship with their host. Seed and environment are sources of } \\
\text { microbes that colonize the developing plant; however, the } \\
\text { influence of each remains unclear. Here, using irradiation } \\
\text { combined with surface sterilization to generate near-axenic seed } \\
\text { with disrupted and reduced microbiomes, we contrasted the } \\
\text { colonization potential of seed and soil microbiomes. We } \\
\text { hypothesized that the seed microbiome would be the primary } \\
\text { colonizer of the plant endophytic compartments. Our experimental } \\
\text { design comprised four treatments, using soybean as a model } \\
\text { plant: (i) nearly axenic seed growing in a sterile environment, (ii) } \\
\text { nonaxenic seed inoculated with a microbial soil extract, (iii) nearly } \\
\text { axenic seed inoculated with a microbial seed extract, and (iv) }\end{array}$ & $\begin{array}{l}\text { quantification of the total community, and functional genes } \\
\text { involved in the } \mathrm{N} \text { cycle. Community dynamics were similar for } \\
\text { most treatments within their respective compartments, except for } \\
\text { the soil treatment, where rhizosphere and root microbiomes } \\
\text { differed from other treatments, suggesting that the soil } \\
\text { microbiome colonizes the belowground compartment efficiently } \\
\text { only when the seed microbiome is severely disrupted. For the } \\
\text { shoot, all treatments resembled the seed microbiome treatment, } \\
\text { suggesting that the seedborne bacteria colonize the aboveground } \\
\text { compartment preferentially. Our results highlight the primacy of } \\
\text { the seed microbiome over the soils during early colonization, } \\
\text { putting seed microbes as potential candidates of microbiome } \\
\text { engineering efforts. }\end{array}$ \\
\hline
\end{tabular}

In agricultural ecosystems, plant-associated microbiomes were shown to improve crop yields (Chen et al. 2019), nutrient uptake (Yang et al. 2009), and nutrient leaching reduction (Bender and van der Heijden 2015). In recent years, there have been concerted efforts to manipulate the microbiome of diseased plants to recover and reestablish positive plant-microbe feedback. The identification of a beneficial microbiome community that encompasses microbial genes

\section{'Corresponding author: É. Yergeau; etienne.yergeau@inrs.ca}

Funding: Support was provided by a Discovery Grant from the Natural Sciences and Engineering Research Council of Canada grant RGPIN 2014-05274 to E. Yergeau; and the Innovation and Scarce Skills scholarship from South African National Research Foundation, Fonds de Recherche du Québec, and partly by Foundation ArmandFrappier to I. Moroenyane.

*The $e$-Xtra logo stands for "electronic extra" and indicates that supplementary materials are published online.

The author(s) declare no conflict of interest.

(C) 2021 The American Phytopathological Society enhancing the holobiont fitness (Lemanceau et al. 2017; Toju et al. 2018) and the critical role plant-associated bacterial communities play in mitigating plant pathogen colonization (Durán et al. 2018; Hassani et al. 2018) have been enumerated. Untangling the patterns and mechanisms of colonization within plant microbiome niches is the first step to unlocking holobiont fitness.

On the one hand, plant microbial communities are generally believed to be recruited from the soil and selected to occupy specific spatial and temporal niches (Beckers et al. 2017; Chi et al. 2005; Kandel et al. 2017). Colonization patterns of the endophytic niche space by soilborne microorganisms have been extensively highlighted (Cordovez et al. 2019; Hardoim et al. 2015) along with the evolutionary processes governing assembly of microorganisms in agricultural soils (Jiao et al. 2020) and at the rhizosphere interface (Goss-Souza et al. 2020; Matthews et al. 2019). On the other hand, seed are the dispersal unit of the plant, and the innate microbiome plays an integral role in the preservation and germination of the seed. Seedtransmitted bacterial communities of domesticated wheat were shown to be less diverse than their wild counterparts (Özkurt et al. 2020). It was suggested that the seed-transmitted microbiome plays a crucial 
role in priming and niche partitioning (Nelson et al. 2018; Shade et al. 2017; Truyens et al. 2015). In wild acorns, there was clear niche differentiation of both bacterial and fungal seed-transmitted communities and effective differences in colonization patterns of the phyllosphere and root compartments (Abdelfattah et al. 2021). Microbiome tracking experiments suggest that soil and seedborne microbiomes are major sources of phyllosphere communities, with air-transmitted microbiomes playing a minor role (Zhou et al. 2021a), while spatial distance from the air-soil interface was highlighted as a limiting factor in the successful colonization of the phyllosphere by soilborne microbes (Zhou et al. 2021b). Evidence of the role of the seed microbiome in characterizing colonization patterns of plant microbiomes is slowly emerging but its importance relative to the soil microbiome remains known.

A comprehensive seed survey of annual wild plants highlighted the fact that photosynthetic pathways are a strong discriminatory axis for the endophytic communities that are characterized by specialized taxa from the phylum Firmicutes (Girsowicz et al. 2019). In axenic wheat culture systems, competent seed endophytes were shown to colonize the root and shoot compartment while influencing overall microbiome colonization patterns (Robinson et al. 2016). Wheat seed inoculated with native endophytic bacteria demonstrate an amplified natural priority effect, although not when inoculated with their fungal endophyte partners (Ridout et al. 2019). Microbiome transplant studies emphasized the role of the seed endophytic community in modulating microbiome colonization patterns and highlighted substantial overlap in the seed and shoot endophytic bacterial community composition (Tannenbaum et al. 2020; Wang et al. 2020). These studies demonstrated the hereditability of the seedborne microbial community and highlighted the significance of these transmitted taxa. Inoculation of flowers has been demonstrated as a viable method to incorporate beneficial microbes into seed (Mitter et al. 2017); however, in another study, only a subset of the community was shown to be transmitted (Rodríguez et al. 2020). In beans, there seems to high degree of phylogenetic niche conservatism of bacterial communities across different stages of seed maturations (Chesneau et al. 2020). Indeed, we had previously demonstrated the existence of bacterial temporal niche spaces across various developmental stages of soybean (Moroenyane et al. 2021a). Successful vertical transmission of the endophytic microbiome from the shoot into the rhizosphere appears to occur only in soil with a disrupted microbiome (Luo et al. 2019). This suggests that the establishment of the seed and endophytic microbiomes are not only under plant-mediated selection but also influenced by other ecological factors. Thus, understanding how different microbiome sources influence community colonization patterns is the first step in unlocking the promises of microbiome engineering.

Ecological assembly processes within the soybean rhizosphere are influenced by the microbiome composition (Goss-Souza et al. 2020; Liu et al. 2019), and nutrient acquisition is highly regulated by rhizosphere composition and structure (Bender and van der Heijden 2015; Bender et al. 2015). Nutrient acquisition in soybean is modulated by plant metabolic needs (Copeland et al. 2015; Hara et al. 2019) and there are strong seasonal patterns of nutrient accumulation, with nitrogen $(\mathrm{N})$ being accumulated during the vegetative growth and podfilling developmental stages (Bender et al. 2015). Soil nitrate levels strongly modulate the levels of root colonization and yield is increased when soybean plants utilize both soil nitrate and symbiotic $\mathrm{N}_{2}$ fixation (Gai et al. 2017; Harper 1974). Therefore, for plant nutrition, not only is the taxonomic composition of the recruited microbes important but also their functional capacity. Virtually nothing is known about the functional differences of seed- and soiltransmitted microbiomes, and this follows the trends highlighted above for taxonomic composition.
Here, we were interested in disentangling the influence of different microbiome sources on the composition of the soybean microbiome and its functional consequences, using the $\mathrm{N}$ cycle as an example. We focused on elucidating the role of different microbiome sources (seed and soil) on the plant microbial community diversity, structure, composition, N-cycle function, and abundance under controlled growth chamber experimental conditions. We hypothesized that the innate seed microbiome would be the primary colonizer of the soybean roots and shoots whereas the rhizosphere microbiome would be colonized primarily by the soil microbial communities. We used an innovative approach to generate near-axenic seed and set up a carefully controlled experiment to test our hypothesis.

\section{MATERIALS AND METHODS}

Sand sterilization. To reduce the innate microbial loads and organic matter content (C content), sand was soaked in $10.8 \%$ sodium hypochlorite $(\mathrm{NaClO})$ (Sany, Montreal, Canada) overnight. Bleached sand was thoroughly rinsed with reverse osmosis water to remove all traces of $\mathrm{NaClO}$. Because bleached sand is devoid of any organic compounds and trace elements, the sand was preloaded with iron (III) chloride $\left(\mathrm{FeCl}_{3}\right) \cdot \mathrm{FeCl}_{3}$ is an immobile element in plants, and its deficiency results in decreased production photosynthetic capacity because it affects chlorophyll production. Briefly, $200 \mu \mathrm{M} \mathrm{FeCl}_{3}(\mathrm{pH}$ $=2$ ) was passed through a $850-\mu \mathrm{m}$ sieve containing rinsed sand until the wash-through was below $\mathrm{pH}=4$ (Henry et al. 2006). To this sand, a modified plant nutrient solution (with no nitrogen) was passed through the sand until the $\mathrm{pH}$ of the wash-through was $\geq 5.5$ (Henry et al. 2006). Preliminary tests indicated that plants that were grown in the sand that was not treated with $\mathrm{FeCl}_{3}$ exhibited symptom of chlorosis, as previously reported by Henry et al. (2006). The sand was air dried overnight in a laminar flow hood prior to sterilization in the autoclave at $121^{\circ} \mathrm{C}$ for $15 \mathrm{~min}$ at $101 \mathrm{KPa}$. After this procedure, no PCR-viable DNA could be extracted from the sand.

Seed surface sterilization and irradiation. Soybean seed from cultivar Pioneer: AAC Edwards were imbibed in sterile water for $24 \mathrm{~h}$ at $4^{\circ} \mathrm{C}$ prior to surface sterilization. Seed were placed in 50-ml Falcon tubes with enough $70 \%$ ethanol (EtOH) to cover the seed and mixed by turning the tube for $2 \mathrm{~min}$. Thereafter, the EtOH was poured out and $5 \% \mathrm{NaClO}$ solution was added for $7 \mathrm{~min}$ while gently turning the tube. To remove all traces of $\mathrm{NaClO}$ solution, the seed were rinsed excessively with sterilized water. The seed were then air dried in an open petri dish and, when moderately dry, the petri dishes were sealed. All works were performed under sterile conditions in laminar flow hoods. Once surface sterile seed broke dormancy, seed were exposed to a low dosage of X-ray irradiation at 40 Gy in Rad Source RS 2000 (Buford) in order to reduce the microbial load of seed endophytes. Because bean seed are more highly susceptible to mutation caused by exposure to X-ray irradiation than mature plants or seedlings (Genter and Brown 1941), only seedlings (once primary root had emerged and was growing) were exposed to $\mathrm{X}$-ray irradiation treatment and not the seed, and at a dosage previously shown to not cause any phenotypic abnormalities to plants (Zappala et al. 2013). In preliminary studies, it was determined that 40 Gy was the most conservative upper limit to which we could expose the seedlings before abnormalities were observed (Supplementary Fig. S1). Overall, there were no observed abnormalities in all of the seedlings used in all treatments.

Experimental treatments. All plants were grown in sterile sand for 14 days and supplemented with $5 \mathrm{ml}$ of filter-sterile nutrient solution. As detailed above, seed were surface sterilized and irradiated prior to the commencement of the experiment, apart from the "positive" treatment. In total, there were four treatments: positive (surface sterile and nonirradiated seed + soil inoculum), negative 
(surface sterile and irradiated seed), seed (surface sterile and irradiated seed + endophytic seed inoculum), and soil (surface sterile and irradiated seed + soil inoculum). We used soil extract as an inoculum instead of soil because seedlings emerging from surface-sterilized seed that were in contact with real soil were rapidly killed by fungal pathogens. To make it more comparable, we also decided to use an endophytic seed inoculum. The endophytic seed inoculum was prepared by crushing five surface sterile seed with a sterile pestle and mortar and suspending them in sterile water. The soil inoculum was prepared by suspending $20 \mathrm{~g}$ of soil in $100 \mathrm{ml}$ of sterile water. For both inocula, the suspension was vigorously vortexed, and $1 \mathrm{ml}$ of the suspension was added to corresponding containers at the beginning of the experiment. The soil used was collected in summer 2019 at the Institut National de la Recherche Scientifique (Laval, QC, Canada) from control unplanted plots of an experimental field that had been plowed for the first time in 2016, with no history of agricultural practice for at least 20 years prior to that. Soils that have a history of agricultural practice drastically regulate the composition of free-living microbial communities and change abundance of microbial N-cycling genes (Mendes et al. 2015; Merloti et al. 2019). Here, we wanted to discern whether microorganisms from agricultural naïve soils had the potential to alter soybean colonization patterns and abundance of $\mathrm{N}$-cycling genes. Soil edaphic properties were previously measured by AgroEnviro Lab (La Pocatiere, QC, Canada) and revealed an average $\mathrm{pH}$ of 7.2, $\mathrm{P}$ concentration of $193 \mathrm{~kg} / \mathrm{ha}$, total $\mathrm{N} 0.15 \%, \mathrm{C} / \mathrm{N}$ of 13.1 , density of $1.16 \mathrm{~g} / \mathrm{cm}^{3}$, and porosity of $55.14 \%$; other properties are reported in Supplementary Table S2.

Plant growth conditions and sampling. Seedlings were grown with an 18 -h photoperiod at $25^{\circ} \mathrm{C}$ followed by a 6-h dark period at $20^{\circ} \mathrm{C}$ in a Conviron growth chamber (Winnipeg, MB, Canada). To ensure that plants were not nutrient stressed, containers were supplied with $5 \mathrm{ml}$ of modified Hoagland's plant nutrient solution at the beginning of the experiment, as suggested by Moscatiello et al. (2013). The nutrient solution was filter sterilized at the moment of application. Plants were grown in sterilized PLANTCON plant tissue culture containers (Fisher Scientific, Waltham, MA, U.S.A.) until the vegetative growth developmental stage (V1). Samples were collected from the rhizosphere (sand that directly attached to the root surface), roots, and shoots (leaves and stem). Fresh weight of sampled tissue was recorded and placed in sterile, 50-ml clear polyethylene Falcon test tubes (Tewksbury) in a sterile laminar flow hood. Sampled plant tissue was crushed in liquid nitrogen using a sterile pestle and mortar. For each sample, $0.25 \mathrm{~g}$ of the crushed tissue or $0.25 \mathrm{~g}$ of rhizosphere soil was added to the bead tubes from the Qiagen Power Soil DNA kit (Hilden, Germany) and DNA was extracted following the manufacturer's instructions, except that DNA was eluted in $50 \mu \mathrm{l}$.

Quantitative real-time PCR. Key microbial functional genes involved in the nitrogen cycle were assessed using various primer pairs. Primers amoA1- $\mathrm{f}^{*}\left(5^{\prime}\right.$-GGGGHTTYTACTGGTGGT-3') and amoA2-r (5'-CCCCTCKGSAAAGCCTTCTTC-3') (Levy-Booth et al. 2014), and crenamoA23-f (5'-ATGGTCTGGCTWAGA CG- $\left.3^{\prime}\right)$ and crenamoA616- $r$ ( $5^{\prime}$-GCCATCCATCTGTA-3') (Tourna et al. 2008), targeted the gene encoding for the bacterial and archaeal versions, respectively, of the ammonia monooxygenase subunit A gene $(a m o A)$ involved in the oxidation of ammonia to hydroxylamine during the first step of nitrification. Primers nirK876- $f *$ (5' - ATYGGC CAYGGCGA-3') and nirK1040-r (5'-GCCTCGATCAGRTTRT GGTT-3') (Henry et al. 2004) targeted the gene encoding the copper-containing nitrite reductase (nirK) involved in the reduction of nitrite to nitric oxide during denitrification. Primers PoI-f* (5' $-\mathrm{TG}$ CGAYCCSAARGCBGACTC-3') and PoI-r (5'-ATSGCCATCA TYTCRCCGGA-3') (Poly et al. 2001) targeted the gene encoding the component 2 of the nitrogenase (nifH) involved in the fixation of atmospheric nitrogen to ammonia. Primers F1norA-f*
(5'-CAGACCGACGTGTGCGAAAG-3') and R2norA-r (5' -TCCA CAAGGAACGGAAGGTC-3') (Attard et al. 2010) targeted the gene encoding for the nitrite oxidoreductase $(n x R)$ that oxidize nitrate to nitrite during the second step of nitrification. Total bacterial and archaeal 16S ribosomal RNA (rRNA) gene abundance was estimated using primers 520F (5'-AGCAGCCGCGGTAAT-3') and 799R (5'-CAGGGTATCTAATCCTGTT-3') (Edwards et al. 2008). For all genes, standards and quantitative PCR (qPCR) protocols were prepared and followed as previously described by Yergeau et al. (2020). Briefly, the standard curve was constructed from serially diluted linearized plasmids $\left(10^{8}\right.$ to $10^{2}$ copies/ $\left.\mu \mathrm{l}\right)$ and the iTaq universal SYBRGreen kit was used following the manufacturer's protocol (Bio-Rad Laboratories Inc., Hercules, CA, U.S.A.). All qPCR assays were performed on a Rotor-Gene 6000 and the data were analyzed using the Rotor-Gene 7.1 software (Corbett Research Biosciences, Sydney, NSW, Australia).

16S rRNA gene amplicon sequencing. Extracted DNA was used to construct sequencing libraries according to Illumina's " $16 \mathrm{~S}$ Metagenomic Sequencing Library Preparation" guide (part number 15044223 Rev. B), with the exception of using Qiagen HotStar MasterMix for the first PCR (amplicon PCR) and halving reagent volumes for the second PCR (index PCR). The first PCR (amplicon PCR) was carried out for 25 cycles with annealing temperatures of $55^{\circ} \mathrm{C}$ using the primers 520F and 799R (Edwards et al. 2008), resulting in amplicons of an average length of $280 \mathrm{bp}$. The commercial microbial community standard was from ZymoBIOMICS (product D6300; Zymo Research Corporation, Irvine, CA, U.S.A.); negative PCR and a blank from DNA extraction kit were used as internal standards. This primer pair targeted the bacterial or archaeal V4 hypervariable regions of the 16S rRNA gene and were shown to exclude chloroplast sequences (Edwards et al. 2008). The resulting amplicons were pooled together and sequenced at the Centre d'expertise et de services Génome Québec (Montréal, QC, Canada). Diluted, pooled samples were loaded on an Illumina MiSeq and sequenced using a 500-cycle (paired-end sequencing configuration of $2 \times 250 \mathrm{bp}$ ) MiSeq Reagent Kit v3. The total number of sequences per sample is reported in Supplementary Table S1.

Sequencing data were analyzed using AmpliconTagger (Tremblay and Yergeau 2019) Briefly, raw reads were scanned for sequencing adapters and PhiX spike-in sequences. We removed single-end reads that failed to meet one of these conditions: having average quality Phred score $<25$; having 30 bases of quality Phred score $<15$; or having 1 or more undefined bases $(\mathrm{N})$. The remaining sequences were processed for generating exact sequence variants (ESVs) (DADA2 v1.12.1) (Callahan et al. 2016). Because the quality filtering step was performed in a separate upstream step, we used more lenient parameters for the dada2 workflow, summarized as follows: filterAndTrim $(\operatorname{maxEE}=2$, truncQ $=0, \operatorname{maxN}=0, \min Q=0)$. Errors were learned using the learnErrors(nbases $=1 \mathrm{e} 8$ ) function for both forward and reverse filtered reads. Reads were then merged using the mergePairs $($ minOverlap $=12$, maxMismatch $=0$ ) function. Chimeras were removed with DADA2's internal removeBimeraDeNovo (method $=$ 'consensus') method followed by UCHIME reference (Edgar et al. 2011). ESVs were assigned a taxonomic lineage with the RDP classifier (Wang et al. 2007) using the Silva release 128 database (Quast et al. 2013) supplemented with eukaryotic sequences from the Silva database and a customized set of mitochondria, plasmid, and bacterial 16S sequences (see the AmpliconTagger databases: https://zenodo.org/record/3560150). The RDP classifier gave a score (0 to 1) to each taxonomic depth of each ESV. For each ESV, the taxonomic lineage was reconstructed by keeping only the taxa that had a score $\geq 0.5$. Taxonomic lineages were combined with the cluster abundance matrix obtained above to generate a raw ESV table. From that raw ESV table, an ESV table containing only bacterial 
organisms was generated. Five hundred 1,000-read rarefactions were then performed on this latter ESV table and the average number of reads of each ESV of each sample was then computed to obtain a consensus rarefied ESV table. ESVs pointing to nonbacterial taxa were removed. The ESVs detected in the negative and extraction kit controls were filtered out of the resultant ESV table, which was then used in all subsequent analyses.

$\boldsymbol{\alpha}$ Diversity patterns. The ESV abundance table was normalized such that the summed relative abundance of all ESVs of each sample $=1$. All statistical analyses were performed using $\mathrm{R}$ version 3.5.0 $(\mathrm{R}$ Core Team 2020) unless otherwise stated. Taxon accumulation curves were constructed to determine whether the sampling effort (number of ESV as a function of the number of samples) could recover most of the taxa, while the Preston log-normal curve was used to evaluate the estimated richness and occurrence of rare taxa across all samples (the presence of a normal distribution indicates a higher probability of rare taxa being represented) (Supplementary Fig. S2). An analysis of variance (ANOVA) or Kruskall-Wallis test when data were not normally distributed were performed to evaluate the effects of treatment (negative, positive, seed, and soil) and plant compartment (rhizosphere, root, and shoot) on the number of observed ESVs and Shannon diversity index.

Microbial community structure. Principal coordinate analysis (PCoA) was used to visualize the microbial community structure based on Bray-Curtis dissimilarity. Multivariate dispersions in the community data were evaluated and confirmed using ANOVA with 999 permutations using the vegan package in $\mathrm{R}$ (Oksanen et al. 2013). To assess the relative significances of treatment and plant compartment on the community structure, a permutational multivariate analysis of variance (PERMANOVA) test was performed with 999 permutations. Finally, the 100 most abundant ESVs were used to determine their influence and were visualized using PCoA and heatmaps.

Taxonomic profiles, random forest models, and linear discrimination analysis. The relative abundance of microbial taxa at the phylum and genus taxonomic levels was evaluated across treatments and plant compartments. Ternary plots were generated to visualize enriched taxa across treatments. Random forest algorithms were used to predict the prevalence of taxa associated with each treatment at the genus and ESV taxonomic levels. Random forest prediction was made using the randomForest algorithm (Liaw and Wiener 2002) and implemented on the MicrobiomeAnalysis pipeline (Chong et al. 2020). Differential abundance of genera was explored using the DESeq2 analysis pipeline (Love et al. 2014) and linear discrimination analysis was used to detect differentially abundant bacterial families and genera across all treatments (Chong et al. 2020)

Data availability. Raw sequencing data were submitted to the NCBI Sequence Read Archive under BioProject accession PRJN A697966.

\section{RESULTS}

Plant biomass. All seed broke dormancy at the same time and all seedlings used in the experiment exhibited normal growth, regardless of whether or not they were irradiated. Plant biomass accumulation varied across treatment and within replicates and, although there were no statistical differences across treatments, there were interesting trends (Fig. 1; Supplementary Fig. S2). First, the leaf mass fraction (LMF) highlighted the fraction of the total biomass represented by leaves. The trend that emerged showed that the soil treatment had higher LMF values than other treatments, and the seed treatment had the lowest values. At the end, the positive control showed the highest phenotypic variation (fresh biomass) compared with all treatments (Fig. 1; Supplementary Fig. S2). The seed and negative treatments had similar levels of variation (fresh biomass), whereas the soil treatment showed less variation among replicates in accumulated biomass than other treatments (Fig. 1; Supplementary Fig. S2).

$\boldsymbol{\alpha}$ Diversity. The ESV accumulation curve indicated that a substantial number of microbial ESVs were recovered and the sampling effort was enough (number of recovered ESV as a function of the number of samples sequenced) (Supplementary Fig. S3). Furthermore, the Preston log-normal curve highlighted that the sampling effort was enough to detect most of the rare and low-abundance taxa (Supplementary Fig. S3). The number of observed ESVs in the mock community control corresponded exactly to the number of ESVs expected $(n=10)$, indicating that the read processing resulted in an accurate picture of the microbial communities sequenced. The measured $\alpha$ diversity indices were all significantly influenced by treatment and plant compartments. Overall, there were significant differences between treatments only in the belowground compartments and not in the shoot. The total number of observed ESVs was significantly influenced by treatment $\left(\chi^{2}=19.01, P<0.001\right)$ (Fig. 2A) and plant compartment $\left(\chi^{2}=34.27, P<0.001\right)$ (Fig. 2A). The soil treatment had the highest abundance of ESVs in the rhizosphere and root compartments, while the seed treatment had the second-highest abundance of ESVs in the root compartment (Fig. 2A). Shannon diversity index was significantly influenced by treatment $\left(\chi^{2}=19.97, P<\right.$ 0.001) (Fig. 2B) and plant compartment $\left(\chi^{2}=40.25, P<0.001\right)$ (Fig. 2B). At the rhizosphere, the soil and seed treatments had higher diversity levels, whereas only in the rhizosphere did the soil treatment significantly differ from all other treatments (Fig. 2B).

Microbial community structure. Bray-Curtis dissimilarity was used to infer community $\beta$ diversity patterns and structure. First, homogeneity and multivariate dispersion analysis was used to determine the relative influence of between community composition (treatment variation) and within community composition (variation within replicates) on overall community structure. Community structure was influenced by differences in composition, with treatment and compartment having significant effects. When considering treatment, multivariate dispersion analysis indicated that there were detectable differences in multivariate dispersion across treatments $(F$ value $=$ 16.81, $P<0.001$ ), and pairwise comparison indicated that the soil and seed inoculum were significantly different in all treatments ( $P<0.05$, ANOVA; Tukey's honestly significant difference). However, there were no discernible differences across the four main treatments (negative, positive, seed, and soil), indicating that difference in structure between treatments was a result of community structure and not differences in composition. PCoA was used to evaluate and visualize the community structure. Treatment and plant compartments significantly influenced community structure (Table 1, PERMANOVA). There was a clear separation of samples by plant compartments along the second PCoA axis, and the first PCoA axis separated samples by treatment, with the rhizosphere and root communities of the soil treatment being divergent from all others (Fig. 2C).

Taxonomic composition. Overall, there were variations in the relative abundance of dominant phyla across treatment and plant compartments (Fig. 2D; Supplementary Fig. S4). At the phylum level, Proteobacteria was the dominant phylum across all treatments and plant compartments. At the rhizosphere interface, the soil treatment had a higher relative abundance of rare taxa (relative abundance < $1 \%$ ) in the rhizosphere but not in the root compartment (Fig. 2D). The relative abundance of Gammaproteobacteria was consistently higher in the shoot compartment across all treatments (Fig. 2D). Overall, ternary plots highlighted that the soil treatment had increased occurrence and abundance of ESVs across all treatments (Fig. 3). The soil treatment occurrence and distribution of ESVs were mainly along the root-rhizosphere axis across all treatments (Fig. 3D), while the seed treatment had taxa that were enriched along the root-shoot axis (Fig. 3C). Across all treatments, ESVs belonging to 
Gammaproteobacteria were enriched in the shoot compartment (Fig. 3). Across all treatments, ESVs belonging to Firmicutes and Betaproteobacteria were enriched along the root-rhizosphere axis whereas, in the seed treatment, a proportion of those Betaproteobacteria were enriched along the root-shoot axis (Fig. 3).

Overall, the relative abundance of the most abundant genera was influenced by treatment and plant compartment (Supplementary Fig. S5). Apart from Sphingomonas, all other abundant genera appeared to be constitutively recruited from the seed inoculum or part of the seedborne community (Fig. 4; Supplementary Fig. S6). The relative abundance of Chryseobacterium $\left(\chi^{2}=31.04^{* * *}\right)$, Methylobacterium $\left(\chi^{2}=26.67^{* *}\right)$, Pantoea $\left(\chi^{2}=15.84^{* *}\right)$, and Pseudomonadaceae $\left(\chi^{2}=11.12^{*}\right)$ was influenced by treatment (Fig. 4$)$. The relative abundance of these taxa was consistently lower for the soil treatment in the rhizosphere and root compartments. Differential abundance analysis of these taxa highlighted that these dominant taxa were significantly more prevalent across all treatments, except for the soil inoculum (Fig. 4; Supplementary Fig. S5). For the rest, plant compartment significantly influenced the relative abundance of Paenibacillus $\left(\chi^{2}=41.93^{* * *}\right)$, Sphingomonas $\left(\chi^{2}=19.07^{* * *}\right.$; treatment $\left.\chi^{2}=19.15^{* * *}\right)$, Stenotrophomonas $\left(\chi^{2}=11.04^{*}\right.$; treatment $\left.\chi^{2}=22.35^{* * *}\right)$, and Burkholderia.Paraburkholderia $\left(\chi^{2}=35.56^{* * *}\right.$; treatment $\chi^{2}=13.16^{*}$ ) (Supplementary Fig. S4). Of these, the relative abundance of Paenibacillus was lowest in the soil treatment and highest in the seed treatment within the rhizosphere (Supplementary Fig. S5). Linear discrimination analysis indicated that most genera or families were significantly enriched in the soil inoculum and soil treatment (Fig. 5; Supplementary Fig. S7; Supplementary Table S3). Finally, the abundance of the nitrogen-fixing genus Rhizobium was significantly enriched in the soil and soil inoculum treatments (Supplementary Fig. S8).

Functional gene abundance. Treatment and plant compartment significantly influenced the abundance of all measured functional genes along with the 16S rRNA gene (Fig. 6). Of all the measured microbial functional genes, the abundance of the bacterial ammonia monooxygenase subunit A gene (amoA) and the archaeal amoA significantly varied in the root compartment (Fig. 6) while the abundance of nitrite oxidoreductase gene $(n x R)$ significantly varied in both rhizosphere and shoot compartments (Fig. 6) $\left(\chi^{2}=36.36^{* * *}\right)$ The abundance of $n x R$ was significantly higher in the soil treatment (Fig. 6) $\left(\chi^{2}=18.23^{* *}\right)$. The abundance of the gene coding for component 2 of the nitrogenase gene (nifH) was not influenced by treatment but there was an influence of compartment (Fig. 6) $\left(\chi^{2}=51.60^{* * *}\right)$. The abundance of the $\mathrm{Cu}$-containing nitrite reductase gene (nirK) was significantly higher in the root compartment across all treatments (Fig. 6). The abundance of the 16S rRNA gene (as a proxy for
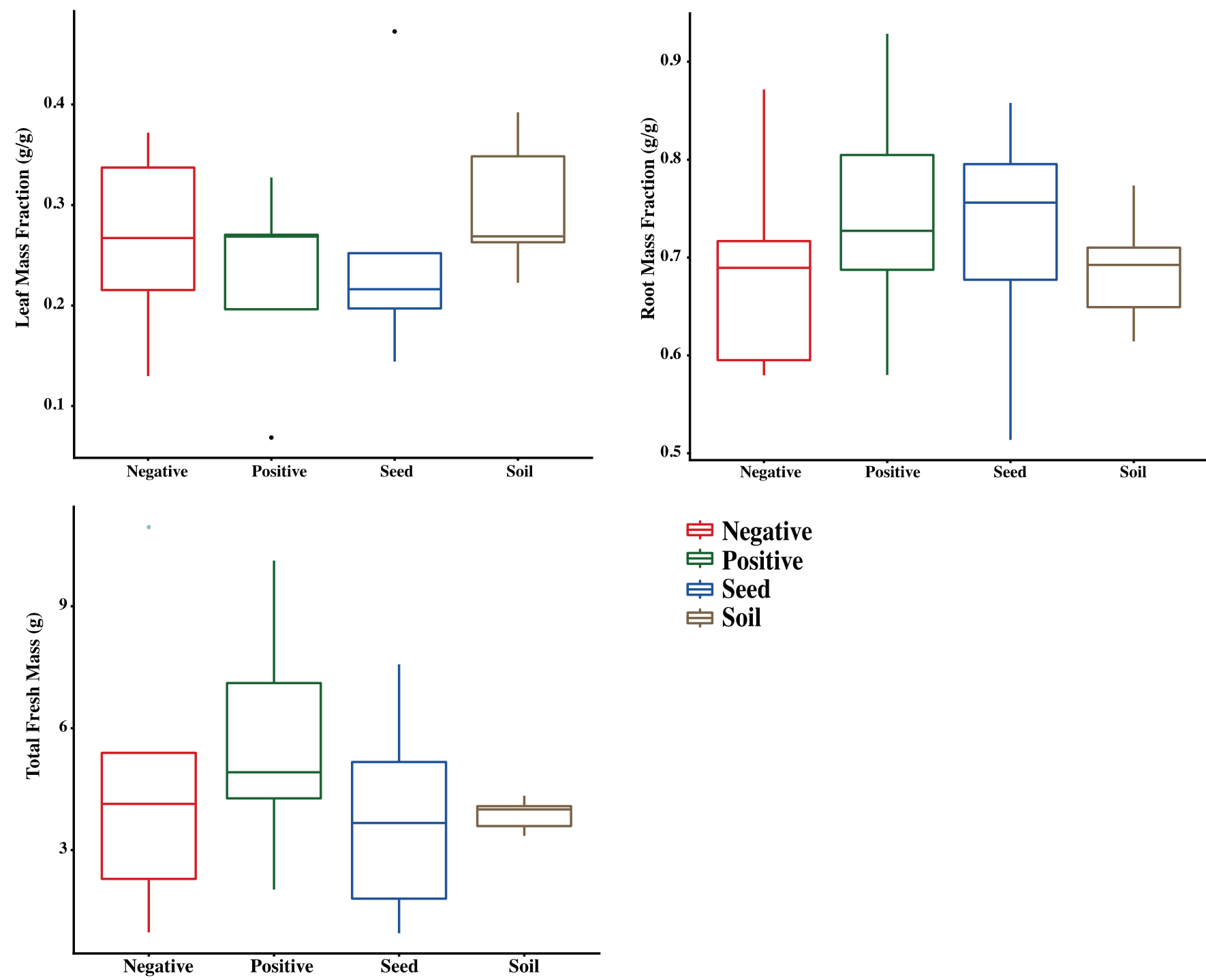

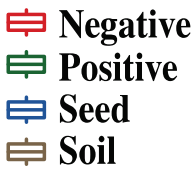

Fig. 1. Plant biomass allocation across different treatments indicating root mass fraction, leaf mass fraction, and total fresh biomass. 
bacterial and archaeal abundance) was marginally significantly affected by the treatment (Fig. 6) $\left(\chi^{2}=11.15^{*}\right)$. In the rhizosphere, the ratios in the abundance between $\mathrm{N}$-cycling genes (nifH, nirK, and $n x R$ ) and the 16S rRNA gene were all significantly higher in the soil treatment (Fig. 7) $(P<0.05)$.

Abundant microbial ESVs. The community structure and composition of the 100 most abundant ESVs across the four main treatments were visualized using PCoA of Bray-Curtis dissimilarity, and random forest analyses were used to predict taxa that were associated with each treatment. Overall, the community structure of the 100 most abundant ESVs was similar to the overall community structure (Supplementary Fig. S9). Treatment and plant compartments significantly influenced community structure (Table 1; PERMANOVA). A heatmap of the 100 most abundant ESVs (genus-level taxonomic affiliation) highlighted the fact that various clusters of taxa that were enriched in the soil and seed treatments (Supplementary Fig. S10). Specifically, there were ESVs that were enriched in both the rhizosphere and root compartments, whereas another cluster highlighted ESVs that were only enriched in the rhizosphere. Taxa such as Paenarthobacter and Paenibacillus that were predicted to be enriched in the seed treatment using random forest analyses (Supplementary Figs. S9 and S10) also formed clusters of enriched ESVs in the heatmap (Supplementary Fig. S10). Similarly, Fluviicola, Dydobacter, and Peridibacter were predicted to be associated with the soil treatment and were part of the cluster that was only enriched in the rhizosphere compartment (Supplementary Figs. S9 and S10).

\section{DISCUSSION}

The goal of our study was to contrast the seed and soil routes for plant microbial colonization. We had hypothesized that the seed microbial communities would be the primary colonizers of the plant environment, with a more substantial effect in the shoot and root environments as compared with the rhizosphere. Most of our results pointed out that the soybean plants grown from seed that were surface sterilized and irradiated before being inoculated with a soil microbial extract showed different microbial community diversity, structure, composition, and functions in their roots and rhizosphere at the emergence stage. These differences did not extend to the shoot microbial communities. The soybean plants grown from surface-sterilized and
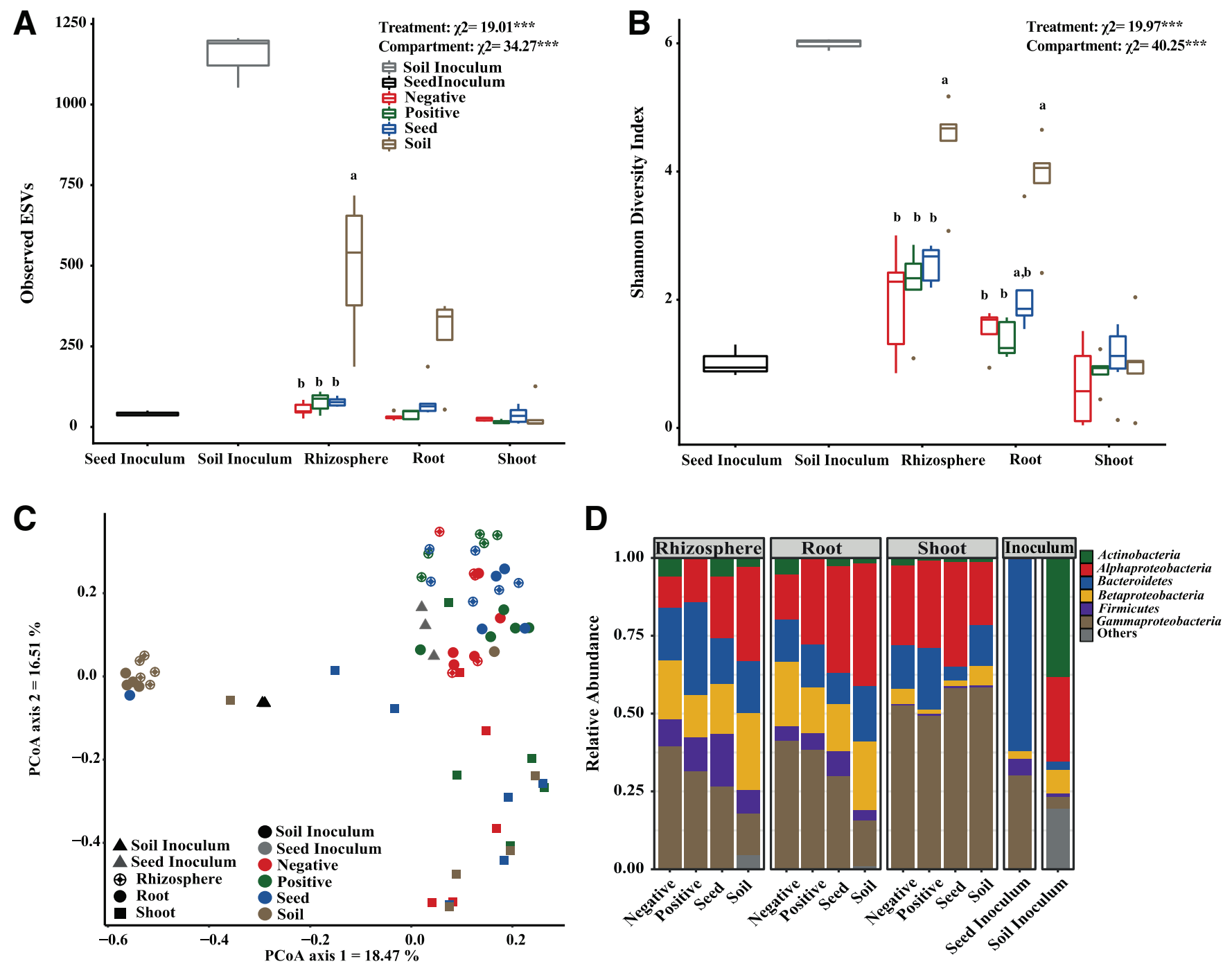

Fig. 2. Microbial diversity patterns indicating bacteria or archaeal diversity influenced by treatment and compartment for $\mathbf{A}$, observed exact sequence variants (ESVs); B, Shannon diversity index; C, principle coordinate analysis (PCoA) of bacterial or archaeal community based on Bray-Curtis dissimilarity; and $\mathbf{D}$, relative abundance of bacterial taxa across plant compartments and treatments. Taxa with relative abundance $<1 \%$ were grouped as "other". Kruskal-Wallis $\chi^{2}$ and $P$ value; superscript letters represent pairwise Wilcox test comparison with Bonferroni correction and asterisks *, **, and $* * *$ indicate $P$ value $<0.05,0.01$, and 0.001 , respectively. 
irradiated seed that were not treated with a soil inoculum developed microbial communities strikingly similar to the ones harbored by the plant grown from surface-sterilized and irradiated seed that were inoculated with a seed extract, or to the ones harbored by plants

TABLE 1

Permutation analysis of variance (PERMANOVA) results indicating the influence of treatment and compartment interaction on microbial community structure

\begin{tabular}{|c|c|c|c|}
\hline \multirow[b]{2}{*}{ Source of variation } & \multicolumn{3}{|c|}{ Bray-Curtis dissimilarity ${ }^{a}$} \\
\hline & MS & $F$ value & $R^{2}$ \\
\hline \multicolumn{4}{|c|}{ Bacterial or archaeal community } \\
\hline Treatment & 0.82 & 6.89 & $0.26^{* * *}$ \\
\hline Compartment & 1.23 & 10.23 & $0.14^{* * *}$ \\
\hline Treatment $\times$ compartment & 0.24 & 2.04 & $0.09^{* *}$ \\
\hline Residuals & 0.11 & - & 0.49 \\
\hline \multicolumn{4}{|c|}{ Top 100 exact sequence variants } \\
\hline Treatment & 0.61 & 6.94 & $0.19^{* * *}$ \\
\hline Compartment & 1.98 & 12.32 & $0.22 * * *$ \\
\hline Treatment $\times$ compartment & 0.17 & 1.99 & $0.11^{* *}$ \\
\hline Residuals & 0.08 & - & 0.47 \\
\hline
\end{tabular}

grown from seed that were not irradiated and inoculated with a soil extract. This suggests that (i) the seed microbial communities were not completely destroyed by our method; (ii) severely disrupted seed microbial communities can recolonize the plant if not in competition with soil microbial communities; (iii) when the seed microbial communities are not disrupted, they have priority over soil microbial communities during plant root and rhizosphere colonization; and (iv) even if the seed microbial communities are disrupted and exposed to soil microbes, they can successfully colonize the aboveground plant compartments.

As such, our hypothesis is confirmed, with the addition that the seedborne microbial communities also appeared to be able to colonize the rhizosphere in the presence of competing soil microbial communities. Even though our results stem from a highly controlled experiment, they are aligned with previous reports that showed that seed microbial communities are the primary source of microorganisms in the plant environment (Chesneau et al. 2020; Rodríguez et al. 2020; Wang et al. 2020).

In soybean, microbiome colonization patterns are modulated by changes in the abiotic environment (Liu et al. 2019; Sanz-Saez et al. 2019), a strong influence of priority effect (Hara et al. 2019), interactions of spatial (plant compartment) and temporal dynamics (developmental stage) (Miller and Roy 1982; Moroenyane et al. 2021b), and plant-mediated selection, with dispersal limitations influencing community assembly processes (Moroenyane et al. 2021a). Here, we sought to highlight colonization patterns of different microbiome
A

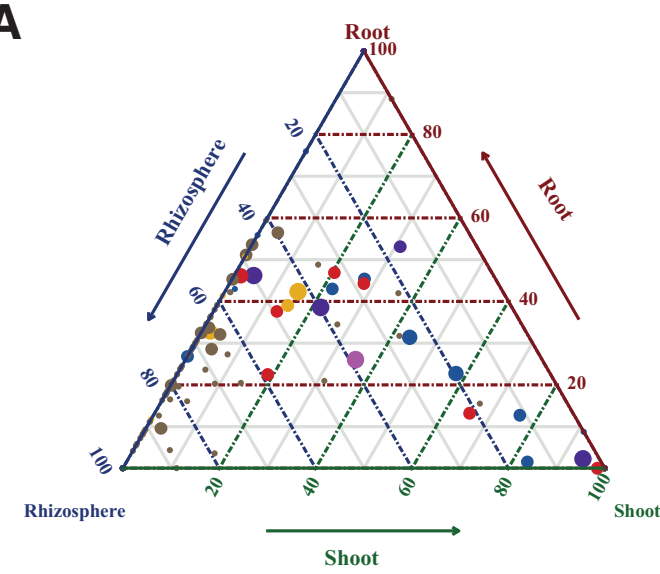

C

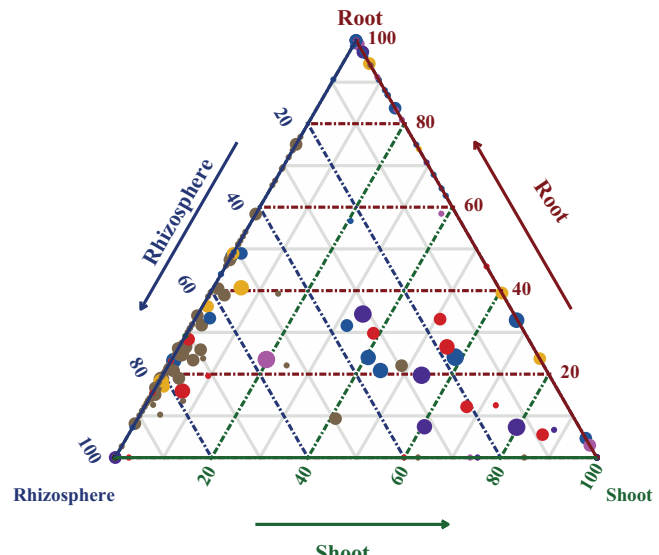

B Rhizosphere

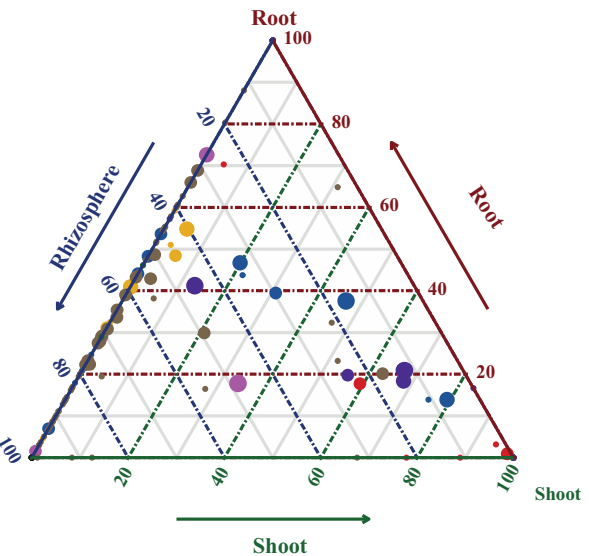

- $0.01-0.1 \%$

- $0.1-1 \%$

- $1-10 \%$

- $10-100 \%$

- Actinobacteria

- Alphaproteobacteria

- Betaproteobacteria

- Gammaproteobacteria

- Firmicutes

- Bacteroidetes

- Gemmatimonadetes

- Other

Shoot

D

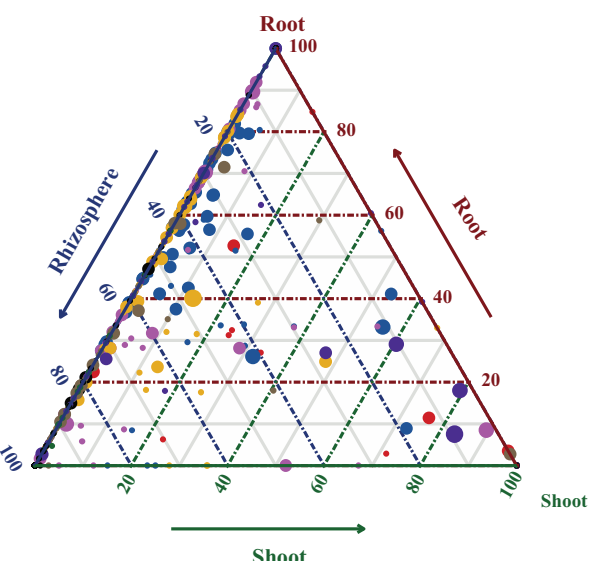

Fig. 3. Ternary plots representing the relative occurrence of exact sequence variants (ESVs) (circles) in A, negative; $\mathbf{B}$, positive; C, seed; and D, soil treatments. Taxa in different plant compartments are colored by their taxonomy at the phylum and class level for Proteobacteria. Size of circle is proportional to mean abundance in the community. 

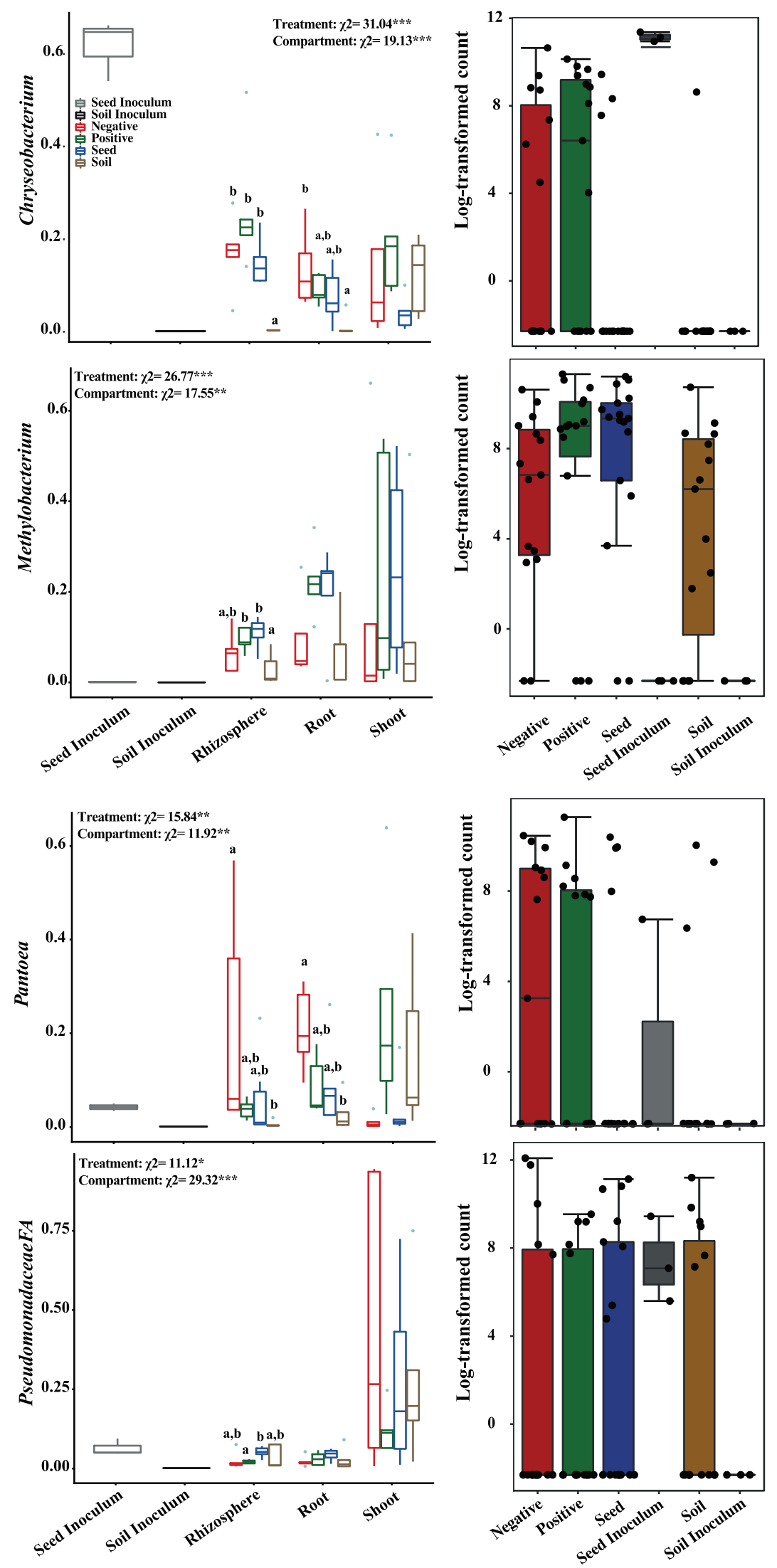

Fig. 4. Left panels: Relative abundance of most abundant genera that were significantly influenced by treatment and compartment (Kruskal-Wallis $\chi^{2}$, $P$ value) and pairwise Wilcox test with Bonferroni correction (asterisks *, **, and *** indicate $P$ value $<0.05,0.01$, and 0.001 , respectively). Right panels: DESeq2 differential abundance analysis of most abundant genera $(P$ value $<0.05)$. 


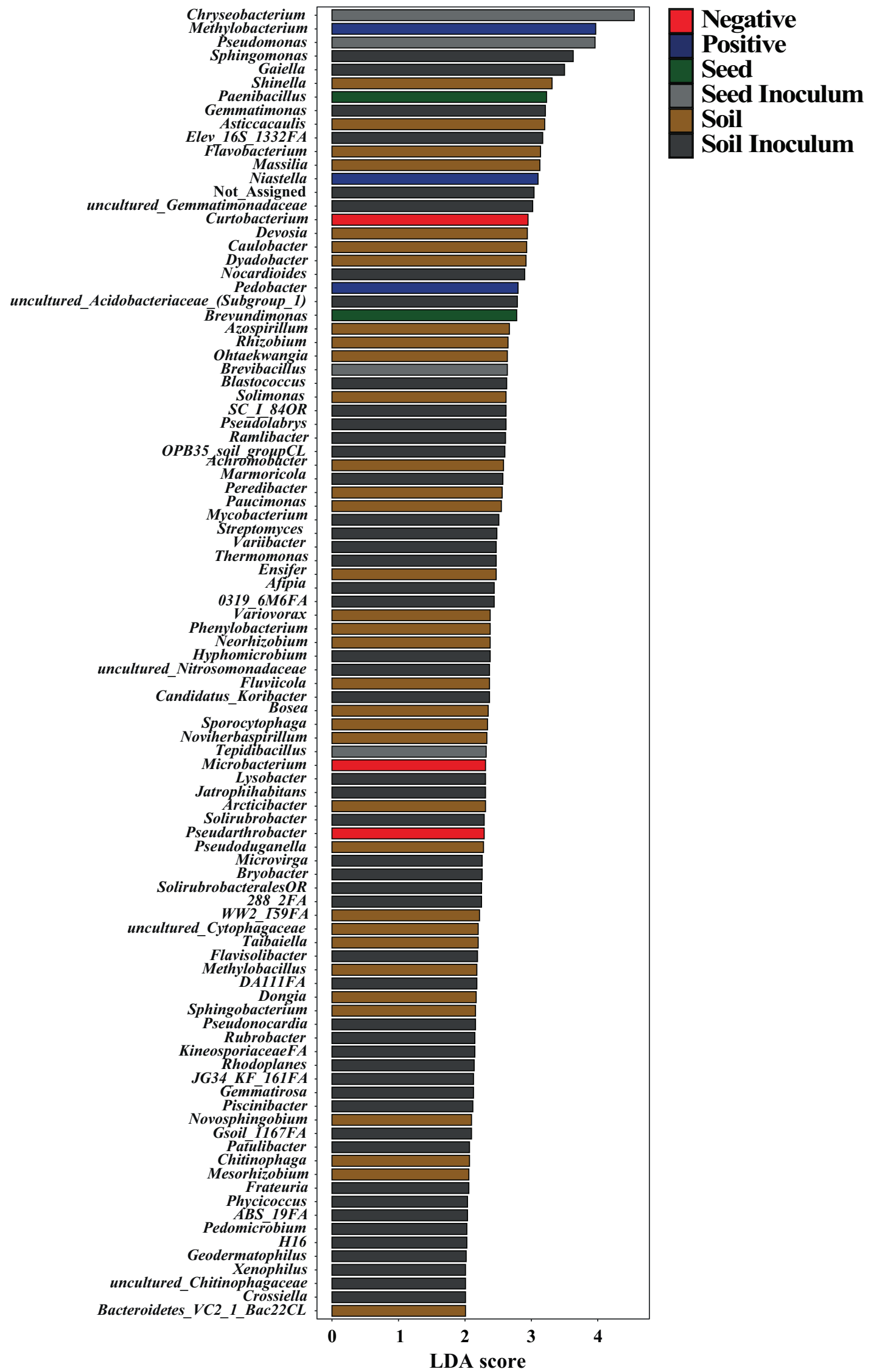

Fig. 5. Linear discriminatory analysis (LDA) indicating differentially abundant genera across all treatments. LDA scores obtained from LEfSE analysis of microbiota across all treatments (LDA effect size $>2$ was used as threshold of LEfSe analysis and $P$ value of 0.05 ). 
sources on near-axenic plants grown in aseptic conditions. Surface sterilization alone has been shown to be effective in the removal of microbial communities in legume seed (Caetano-Anollés et al. 1990), and the use of irradiation to preserve and decontaminate agricultural crops, spices, and meat products is documented (Sommers 2012). However, our fluorescence in-situ hybridization investigation of the irradiated seed indicated the presence of bacterial genomic material within the seed tissue (not shown). Ionizing radiation interacts with DNA by causing irreversible degradation, and there is a positive correlation between dosage and DNA damage (Borgognoni et al. 2017; Moosekian et al. 2012; Stuy 1960). Efficacy is influenced by dosage, the composition of the biological material (density, temperature, $\mathrm{pH}$, and innate gases), and innate microbial composition, with Gram-positive bacteria being more tolerant than Gram-negative bacteria (Moosekian et al. 2012). X-ray irradiation decreased fruitborne microbial communities fivefold in tomato (Mahmoud 2010) and tolerance is a strain-specific physiological trait (Beblo-Vranesevic et al. 2018). It is then possible that the low irradiation dosage administered in the experiment only reduced the microbial loads and did not completely eradicate seedborne microbes.

Surface-sterilized soybean seed harbor microorganisms that proliferate during germination and colonize the plant surface (CaetanoAnollés et al. 1990). Plant-growth-promoting bacteria from seed are considered to be the early colonizers of the spermosphere; in fact,
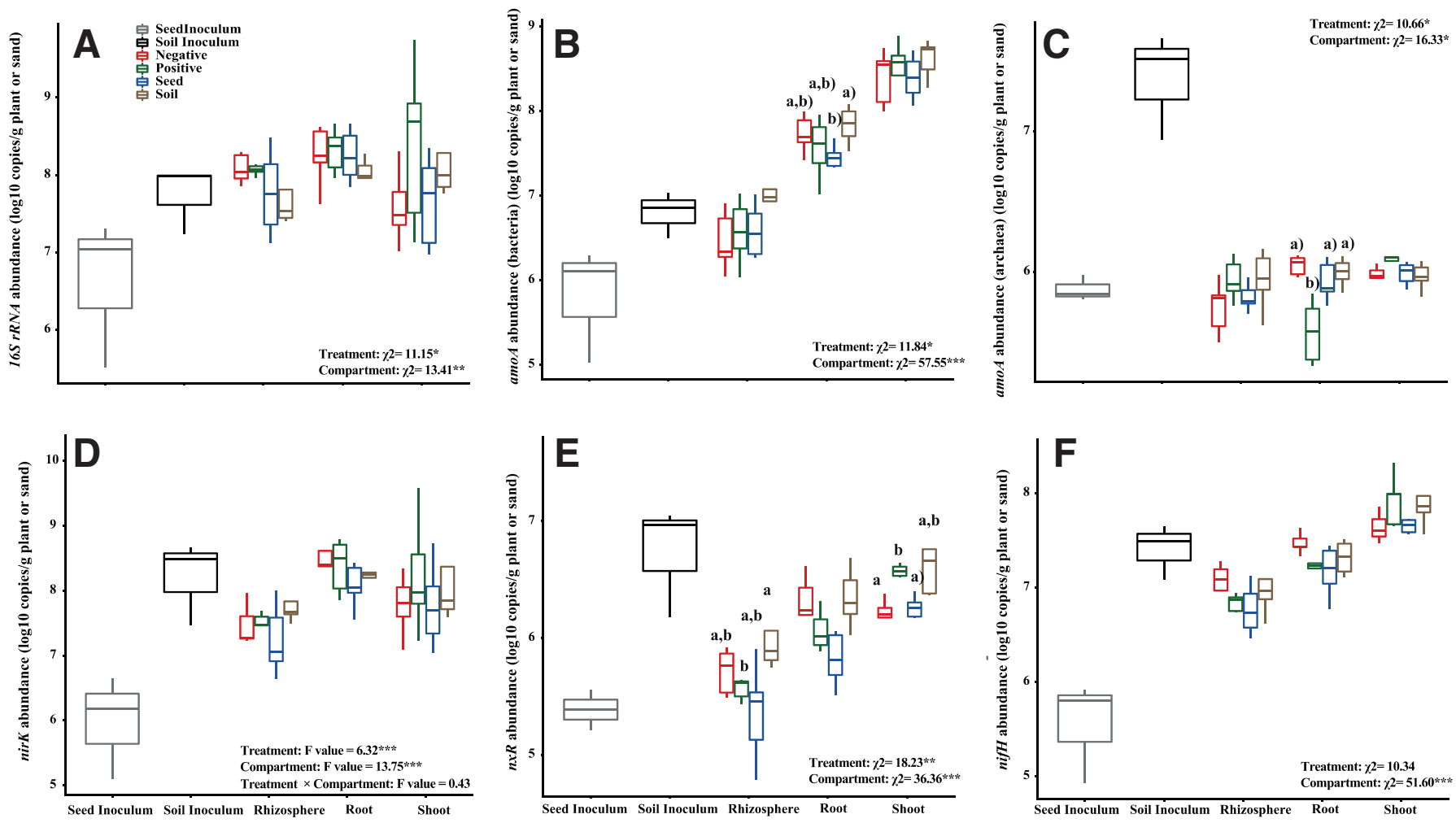

Fig. 6. Abundance of microbial functions diversity influenced by treatment and compartment for A, 16S ribosomal RNA (rRNA) gene, B, amoA bacteria, C, amoA archaea, D, nirK, E, $n x R$, and F, nifH. Kruskal-Wallis $\chi^{2}, P$ value; and analysis of variance $F$ value. Asterisks *, **, and *** indicate $P$ value $<0.05,0.01$, and 0.001 , respectively. Letters denote Tukey's honestly significant difference pairwise or Wilcox test comparison with Bonferroni correction.
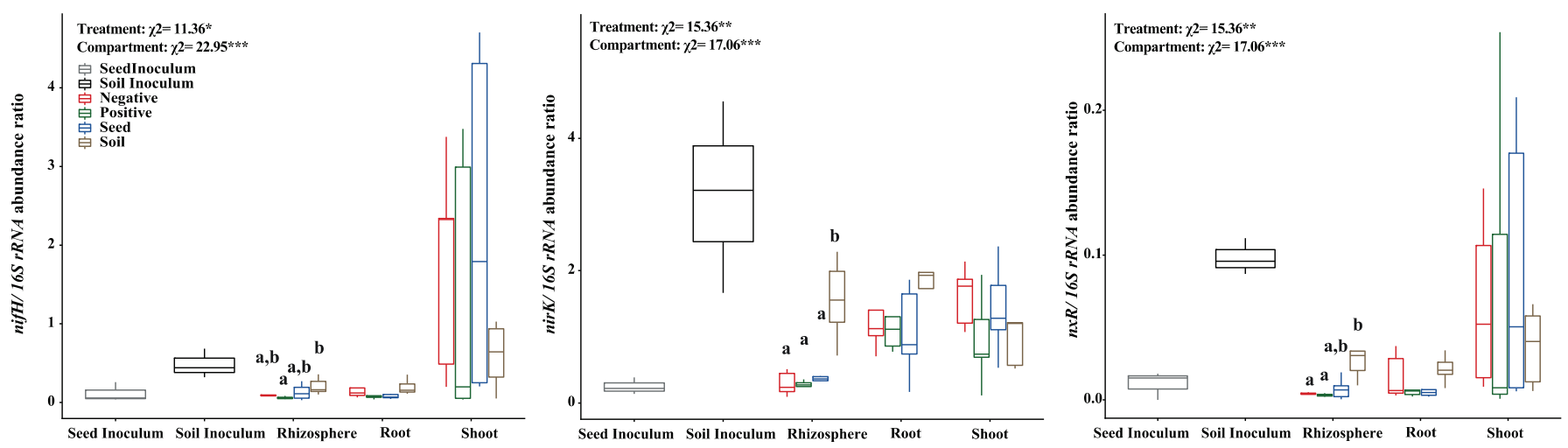

Fig. 7. Abundance ratios of N-cycling genes and 16S ribosomal RNA (rRNA) genes by treatment and compartment for the nifH/16S rRNA gene, nirK/ 16S rRNA gene, and $n x R / 16 S$ rRNA gene. Kruskal-Wallis $\chi^{2}$; asterisks $*,{ }^{* *}$, and $* * *$ indicate $P$ value $<0.05,0.01$, and 0.001 , respectively. Letters denote pairwise Wilcox test with Bonferroni correction. 
endophytic bacteria from Eucalyptus seed that were tagged with the gfp gene have been recovered from the phyllosphere of seedlings (Ferreira et al. 2008). Similarly, known soybean seed growthpromoting bacteria tagged with the $g f p$ gene were shown to colonize the inner compartment of roots and shoots (Batista et al. 2018). These studies highlighted the fact that bacterial taxa from seed can easily colonize the shoots and roots of host plants but also the intercellular spaces. The intercellular space is enriched with carbohydrates, amino acids, and inorganic nutrients needed by the bacterial endosymbiont. Endophytic microbes have adapted to a nutrient-replete environment that has relatively subdued levels of interspecific competition when compared with the rhizosphere or phyllosphere counterpart communities (Bacon and Hinton 2007; Sturz et al. 2000). However, this view has been challenged, because most endophytic microbes are facultative symbionts and compete with soilborne microbes at the rhizosphere interface prior to entry into the plant (Compant et al. 2010). Here, we propose that, at the rhizosphere interface, seedborne microorganisms are capable of outcompeting members of the rhizosphere to quickly colonize the internal plant compartments, and even the rhizosphere itself. Furthermore, even when this innate endophytic seed microbiome is severely disrupted, it is still capable of colonizing the rhizosphere and endosphere in the absence of competition from the rhizosphere microbiome.

Microorganisms involved in the inorganic $\mathrm{N}$ cycle have a key role in changing the availability of soil $\mathrm{N}$ for plant uptake; therefore, we selected this group as a model functional group to test the effects of our treatments. Interestingly, the abundance of the two bacterial genes involved in nitrification (bacterial $a m o A$ and $n x R$ ) were the only ones significantly influenced by the treatments, being generally more abundant under the soil treatment. This was probably linked to their higher abundance in the soil inoculum, as highlighted in linear discriminatory analysis (LDA) for the Nitrosomonadaceae, an ammoniaoxidizer bacterial family. However, we could detect all of the functional genes in all of the treatments even in the negative treatment, suggesting that these functional groups can be seed transmitted. Because nitrification transforms the more energetically favorable ammonia in the less energetically favorable nitrate (Moreau et al. 2019), and that denitrification results in a net loss of nitrogen from the soil, the presence of these functional genes could negatively impact plant $\mathrm{N}$ nutrition, which makes their transmission through seed intriguing. Interestingly, under our experimental setup, many of the genes were significantly more abundant in root and shoot samples and less abundant in the rhizosphere. We found that an increased abundance of nitrite-oxidizing bacterial in the rhizosphere of plants of the soil treatment indicated potential increased production of nitrate $\left(\mathrm{NO}^{-3}\right)$, which is shown to be the preferred form of $\mathrm{N}$ for legumes (Cui et al. 2017; Liu et al. 2018), despite uptake of $\mathrm{NO}^{-3}$ being an energy exhaustive process for the plant (Moreau et al. 2019). This suggests that exposure of seed to the soil microbiome might have some positive effects on plant nutrition. Equally, the increased abundance of nitrite reductases (nirK) containing bacteria in the rhizosphere of the soil treatment was coherent with the LDA that revealed known denitrifying taxa (e.g., Paucimonas) (Pichinoty et al. 1977) being associated with the soil treatment This suggests that these functional groups, though more abundant in the bulk soil inoculum, have a strong capacity to colonize plants. As for their role inside roots and shoots, we can only speculate that they might take advantage of the inorganic $\mathrm{N}$ in planta as a substrate. The potential presence of fungi in the soil inoculum could have further increased the abundance of denitrifiers in the soil treatment, as previously observed in agricultural fields (Gui et al. 2021; Ma et al. 2017; Xu et al. 2019). Overall, the trends observed in the functional genes were highly similar to the ones observed using taxonomic marker genes, suggesting that the seed microbiome primacy has consequences not only on the microbial community composition of the soybean environment but also on the associated functions.

Interspecific competition and priority effects are pronounced at the rhizosphere interface and influence microbial colonization patterns (Choi et al. 2020; Compant et al. 2010). Our data indicate that the intact seed microbiome has priority and outcompetes the invading soil microbiome, and only when the innate seed microbiome is severely disrupted is there successful colonization of the rhizosphere compartments by the soil microbiome. In axenic turfgrass culture, the extant soil and not the seed microbiome was shown to assert a more substantial influence on the microbiome structure (Doherty et al. 2020). Microbiome transplant studies tend to focus on measuring the performance of native plants grown in sterile soils that are inoculated with a foreign microbiome and reveal intricate plant-soil feedback, where native plant performance is amplified when inoculated with a native soil microbiome (Smith et al. 2018). However, these studies tend to use autoclaved soil or peat to reveal these colonization patterns of the belowground compartment, and such experimental setups cannot highlight the role of the seed microbiome. Our closedchamber and reductionist experimental setup allowed us to discern the nuanced influences of seed and soil microbiomes on plant colonization patterns and highlighted the primacy of the seed microbiome over the soil microbiome.

In the current study, the phyllosphere was significantly different from the other plant compartments, being not influenced by the soil microbiome even when the seed microbiome was severely disrupted. In culture-based experiments, intact seed endophytes influenced secondary colonization of the rhizosphere (Ridout et al. 2019) and phyllosphere compartments (Carlström et al. 2019). Colonization patterns within the phyllosphere microbiome were shown to be dominated by generalist bacterial taxa (Massoni et al. 2020). Our results highlighted that the community composition and structure of the phyllosphere were similar across all treatments, and all communities were dominated by ESVs from same genera. In field conditions, early colonization patterns within the phyllosphere of agricultural crops are driven by recruitment from native soils and the environment (de Souza et al. 2016; Grady et al. 2019), even within soybean (Copeland et al. 2015). Here, we showed that whether or not the recruitment from the environment is impaired, the innate seed microbiome will occupy the endophytic and epiphytic niche spaces of the phyllosphere, emphasizing the nuanced influence of seed microbiomes as potential priority effect agents. Overall, our experiments highlight the idea that inoculation of the rhizosphere is insufficient to drive community change within the phyllosphere, and are in agreement with previous coalescence experiments that demonstrated that the strength of host selection only increases with successive microbiome passages (Morella et al. 2020) and developmental stages (Copeland et al. 2015).

Conclusion. In conclusion, this study provides support for our hypothesis that only when the innate seed microbiome is severely disrupted will the soil microbiome colonize the rhizosphere and influence the microbial community diversity, abundance, and composition at both the functional and taxonomical levels. In all other cases, the seed microbiome has priority over the soil microbiome. We also highlighted the significant differences in the influence of the soil microbiome between aboveground and belowground compartments. Our results are coherent with the hologenome theory of evolution, because a large part of the soybean microbiota appears to be seed transmitted and to have the upper hand over microbes from the environment. Although these results would have to be confirmed in the field, where conditions are more variable and environmental sources more abundant, our results point toward the seed microbiome as the most promising candidate for plant microbiome engineering efforts. 


\section{ACKNOWLEDGMENTS}

We thank the Department of Agriculture and Agri-Food Canada for providing the seed used in the study, and Compute Canada for access to the University of Waterloo's High-Performance Computing infrastructure (Graham system) through a resources allocation granted to E. Yergeau.

\section{LITERATURE CITED}

Abdelfattah, A., Wisniewski, M., Schena, L., and Tack, A. J. M. 2021. Experimental evidence of microbial inheritance in plants and transmission routes from seed to phyllosphere and root. Environ. Microbiol. 23: 2199-2214.

Attard, E., Poly, F., Commeaux, C., Laurent, F., Terada, A., Smets, B. F., Recous, S., and Roux, X. L. 2010. Shifts between Nitrospira-and Nitrobacter-like nitrite oxidizers underlie the response of soil potential nitrite oxidation to changes in tillage practices. Environ. Microbiol. 12: 315-326.

Bacon, C. W., and Hinton, D. M. 2007. Bacterial endophytes: The endophytic niche, its occupants, and its utility. Pages 155-194 in: Plant-Associated Bacteria. S. S. Gnanamanickam, ed. Springer, Dordrecht, The Netherlands.

Batista, B. D., Lacava, P. T., Ferrari, A., Teixeira-Silva, N. S., Bonatelli, M. L., Tsui, S., Mondin, M., Kitajima, E. W., Pereira, J. O., and Azevedo, J. L. 2018. Screening of tropically derived, multi-trait plant growth-promoting rhizobacteria and evaluation of corn and soybean colonization ability. Microbiol. Res. 206:33-42.

Beblo-Vranesevic, K., Bohmeier, M., Perras, A. K., Schwendner, P., Rabbow, E., Moissl-Eichinger, C., Cockell, C. S., Vannier, P., Marteninsson, V. T., Monaghan, E. P., Ehrenfreund, P., Garcia-Descalzo, L., Gómez, F., Malki, M., Amils, R., Gaboyer, F., Westall, F., Cabezas, P., Walter, N., and Rettberg, P. 2018. Lack of correlation of desiccation and radiation tolerance in microorganisms from diverse extreme environments tested under anoxic conditions. FEMS Microbiol. Lett. 365:fny044.

Beckers, B., Op De Beeck, M., Weyens, N., Boerjan, W., and Vangronsveld, J. 2017. Structural variability and niche differentiation in the rhizosphere and endosphere bacterial microbiome of field-grown poplar trees. Microbiome $5: 25$.

Bender, R. R., Haegele, J. W., and Below, F. E. 2015. Nutrient uptake, partitioning, and remobilization in modern soybean varieties. Agron. J. 107: 563-573.

Bender, S. F., and van der Heijden, M. G. A. 2015. Soil biota enhance agricultural sustainability by improving crop yield, nutrient uptake and reducing nitrogen leaching losses. J. Appl. Ecol. 52:228-239.

Borgognoni, F., Vadrucci, M., Bazzano, G., Ferrari, P., Massa, S., Moretti, R., Calvitti, M., Ronsivalle, C., Moriani, A., and Picardi, L. 2017. X-ray sterilization of insects and microorganisms for cultural heritage applications. Nucl. Instrum. Methods Phys. Res. B 406:309-313.

Caetano-Anollés, G., Favelukes, G., and Bauer, W. 1990. Optimization of surface sterilization for legume seed. Crop Sci. 30:708-712.

Callahan, B. J., McMurdie, P. J., Rosen, M. J., Han, A. W., Johnson, A. J., and Holmes, S. P. 2016. DADA2: High-resolution sample inference from Illumina amplicon data. Nat. Methods 13:581-583.

Carlström, C. I., Field, C. M., Bortfeld-Miller, M., Müller, B., Sunagawa, S., and Vorholt, J. A. 2019. Synthetic microbiota reveal priority effects and keystone strains in the Arabidopsis phyllosphere. Nat. Ecol. Evol. 3: 1445-1454.

Chen, S. M., Waghmode, T. R., Sun, R. B., Kuramae, E. E., Hu, C. S., and Liu, B. B. 2019. Root-associated microbiomes of wheat under the combined effect of plant development and nitrogen fertilization. Microbiome 7:136.

Chesneau, G., Torres-Cortes, G., Briand, M., Darrasse, A., Preveaux, A., Marais, C., Jacques, M. A., Shade, A., and Barret, M. 2020. Temporal dynamics of bacterial communities during seed development and maturation. FEMS Microbiol. Ecol. 96:fiaa190.

Chi, F., Shen, S. H., Cheng, H. P., Jing, Y. X., Yanni, Y. G., and Dazzo, F. B. 2005. Ascending migration of endophytic rhizobia, from roots to leaves, inside rice plants and assessment of benefits to rice growth physiology. Appl. Environ. Microbiol. 71:7271-7278.

Choi, K., Choi, J., Lee, P. A., Roy, N., Khan, R., Lee, H. J., Weon, H. Y., Kong, H. G., and Lee, S. W. 2020. Alteration of bacterial wilt resistance in tomato plant by microbiota transplant. Front. Plant Sci. 11:1186.
Chong, J., Liu, P., Zhou, G. Y., and Xia, J. G. 2020. Using MicrobiomeAnalyst for comprehensive statistical, functional, and meta-analysis of microbiome data. Nat. Protoc. 15:799-821.

Compant, S., Clément, C., and Sessitsch, A. 2010. Plant growth-promoting bacteria in the rhizo-and endosphere of plants: Their role, colonization, mechanisms involved and prospects for utilization. Soil Biol. Biochem. 42: 669-678.

Copeland, J. K., Yuan, L. J., Layeghifard, M., Wang, P. W., and Guttman, D. S. 2015. Seasonal community succession of the phyllosphere microbiome. Mol. Plant-Microbe Interact. 28:274-285.

Cordovez, V., Dini-Andreote, F., Carrion, V. J., and Raaijmakers, J. M. 2019. Ecology and evolution of plant microbiomes. Annu. Rev. Microbiol. 73: 69-88.

Cui, J., Yu, C., Qiao, N., Xu, X., Tian, Y., and Ouyang, H. 2017. Plant preference for $\mathrm{NH}_{4}{ }^{+}$versus $\mathrm{NO}_{3}{ }^{-}$at different growth stages in an alpine agroecosystem. Field Crops Res. 201:192-199.

de Souza, R. S. C., Okura, V. K., Armanhi, J. S. L., Jorrín, B., Lozano, N., da Silva, M. J., González-Guerrero, M., de Araújo, L. M., Verza, N. C., Bagheri, H. C., Imperial, J., and Arruda, P. 2016. Unlocking the bacterial and fungal communities assemblages of sugarcane microbiome. Sci. Rep. 6: 28774.

Doherty, J. R., Crouch, J. A., and Roberts, J. A. 2020. Elucidating the influence of resident seed and soil microbiota on the developing creeping bentgrass microbiome. Agrosyst. Geosci. Environ. 3:e20038.

Durán, P., Thiergart, T., Garrido-Oter, R., Agler, M., Kemen, E., SchulzeLefert, P., and Hacquard, S. 2018. Microbial interkingdom interactions in roots promote Arabidopsis survival. Cell 175:973-983.e14.

Edgar, R. C., Haas, B. J., Clemente, J. C., Quince, C., and Knight, R. 2011. UCHIME improves sensitivity and speed of chimera detection. Bioinformatics 27:2194-2200.

Edwards, J. E., Kingston-Smith, A. H., Jimenez, H. R., Huws, S. A., Skot, K. P., Griffith, G. W., McEwan, N. R., and Theodorou, M. K. 2008. Dynamics of initial colonization of nonconserved perennial ryegrass by anaerobic fungi in the bovine rumen. FEMS Microbiol. Ecol. 66:537-545.

Ferreira, A., Quecine, M. C., Lacava, P. T., Oda, S., Azevedo, J. L., and Araujo, W. L. 2008. Diversity of endophytic bacteria from Eucalyptus species seeds and colonization of seedlings by Pantoea agglomerans. FEMS Microbiol. Lett. 287:8-14.

Gai, Z., Zhang, J., and Li, C. 2017. Effects of starter nitrogen fertilizer on soybean root activity, leaf photosynthesis and grain yield. PLoS One 12: $\mathrm{e} 0174841$.

Genter, C. F., and Brown, H. M. 1941. X-ray studies on the field bean. J. Hered. 32:39-44.

Girsowicz, R., Moroenyane, I., and Steinberger, Y. 2019. Bacterial seed endophyte community of annual plants modulated by plant photosynthetic pathways. Microbiol. Res. 223-225:58-62.

Goss-Souza, D., Mendes, L. W., Rodrigues, J. L. M., and Tsai, S. M. 2020. Ecological processes shaping bulk soil and rhizosphere microbiome assembly in a long-term amazon forest-to-agriculture conversion. Microb. Ecol. 79:110-122.

Grady, K. L., Sorensen, J. W., Stopnisek, N., Guittar, J., and Shade, A. 2019. Assembly and seasonality of core phyllosphere microbiota on perennial biofuel crops. Nat. Commun. 10:4135.

Gui, H., Gao, Y., Wang, Z., Shi, L., Yan, K., and Xu, J. 2021. Arbuscular mycorrhizal fungi potentially regulate $\mathrm{N} 2 \mathrm{O}$ emissions from agricultural soils via altered expression of denitrification genes. Sci. Total Environ. 774: 145133.

Hara, S., Matsuda, M., and Minamisawa, K. 2019. Growth stage-dependent bacterial communities in soybean plant tissues: Methylorubrum transiently dominated in the flowering stage of the soybean shoot. Microbes Environ. 34:446-450.

Hardoim, P. R., Van Overbeek, L. S., Berg, G., Pirttilä, A. M., Compant, S., Campisano, A., Döring, M., and Sessitsch, A. 2015. The hidden world within plants: Ecological and evolutionary considerations for defining functioning of microbial endophytes. Microbiol. Mol. Biol. Rev. 79: 293-320.

Harper, J. E. 1974. Soil and symbiotic nitrogen requirements for optimum soybean production. Crop Sci. 14:255-260.

Hassani, M. A., Durán, P., and Hacquard, S. 2018. Microbial interactions within the plant holobiont. Microbiome 6:58.

Henry, A., Doucette, W., Norton, J., Jones, S., Chard, J., and Bugbee, B. 2006. An axenic plant culture system for optimal growth in long-term studies. J. Environ. Qual. 35:590-598. 
Henry, S., Baudoin, E., López-Gutiérrez, J. C., Martin-Laurent, F., Brauman, A., and Philippot, L. 2004. Quantification of denitrifying bacteria in soils by nirK gene targeted real-time PCR. J. Microbiol. Methods 59:327-335.

Jiao, S., Yang, Y. F., Xu, Y. Q., Zhang, J., and Lu, Y. H. 2020. Balance between community assembly processes mediates species coexistence in agricultural soil microbiomes across eastern China. ISME J. 14:202-216.

Kandel, S. L., Joubert, P. M., and Doty, S. L. 2017. Bacterial endophyte colonization and distribution within plants. Microorganisms 5:77.

Lemanceau, P., Blouin, M., Muller, D., and Moenne-Loccoz, Y. 2017. Let the core microbiota be functional. Trends Plant Sci. 22:583-595.

Levy-Booth, D. J., Prescott, C. E., and Grayston, S. J. 2014. Microbial functional genes involved in nitrogen fixation, nitrification and denitrification in forest ecosystems. Soil Biol. Biochem. 75:11-25.

Liaw, A., and Wiener, M. 2002. Classification and regression by randomForest. $\mathrm{R}$ News 2:18-22.

Liu, F., Hewezi, T., Lebeis, S. L., Pantalone, V., Grewal, P. S., and Staton, M. E. 2019. Soil indigenous microbiome and plant genotypes cooperatively modify soybean rhizosphere microbiome assembly. BMC Microbiol. 19:201.

Liu, M., Qiao, N., Zhang, Q., and Xu, X. 2018. Cropping regimes affect $\mathrm{NO}_{3}{ }^{-}$ versus $\mathrm{NH}_{4}{ }^{+}$uptake by Zea mays and Glycine max. Plant Soil 426:241-251.

Love, M. I., Huber, W., and Anders, S. 2014. Moderated estimation of fold change and dispersion for RNA-seq data with DESeq2. Genome Biol. 15:550.

Luo, J. P., Tao, Q., Jupa, R., Liu, Y., Wu, K., Song, Y., Li, J., Huang, Y., Zou, L., Liang, Y., and Li, T. 2019. Role of vertical transmission of shoot endophytes in root-associated microbiome assembly and heavy metal hyperaccumulation in Sedum alfredii. Environ. Sci. Technol. 53:6954-6963.

Ma, S., Shan, J., and Yan, X. 2017. $\mathrm{N}_{2} \mathrm{O}$ emissions dominated by fungi in an intensively managed vegetable field converted from wheat-rice rotation. Appl. Soil Ecol. 116:23-29.

Mahmoud, B. S. 2010. The effects of X-ray radiation on Escherichia coli O157:H7, Listeria monocytogenes, Salmonella enterica and Shigella flexneri inoculated on whole Roma tomatoes. Food Microbiol. 27:1057-1063.

Massoni, J., Bortfeld-Miller, M., Jardillier, L., Salazar, G., Sunagawa, S., and Vorholt, J. A. 2020. Consistent host and organ occupancy of phyllosphere bacteria in a community of wild herbaceous plant species. ISME J. 14: 245-258.

Matthews, A., Pierce, S., Hipperson, H., and Raymond, B. 2019. Rhizobacterial community assembly patterns vary between crop species. Front. Microbiol. 10:581.

Mendes, L. W., Tsai, S. M., Navarrete, A. A., de Hollander, M., van Veen, J. A., and Kuramae, E. E. 2015. Soil-borne microbiome: Linking diversity to function. Microb. Ecol. 70:255-265.

Merloti, L. F., Mendes, L. W., Pedrinho, A., de Souza, L. F., Ferrari, B. M., and Tsai, S. M. 2019. Forest-to-agriculture conversion in Amazon drives soil microbial communities and N-cycle. Soil Biol. Biochem. 137:107567.

Miller, W. A., and Roy, K. W. 1982. Mycoflora of soybean leaves, pods, and seeds in Mississippi. Can. J. Bot. 60:2716-2723.

Mitter, B., Pfaffenbichler, N., Flavell, R., Compant, S., Antonielli, L., Petric, A., Berninger, T., Naveed, M., Sheibani-Tezerji, R., and von Maltzahn, G. 2017. A new approach to modify plant microbiomes and traits by introducing beneficial bacteria at flowering into progeny seeds. Front. Microbiol. 8:11.

Moosekian, S. R., Jeong, S., Marks, B. P., and Ryser, E. T. 2012. X-ray irradiation as a microbial intervention strategy for food. Annu. Rev. Food Sci. Technol. 3:493-510.

Moreau, D., Bardgett, R. D., Finlay, R. D., Jones, D. L., and Philippot, L. 2019. A plant perspective on nitrogen cycling in the rhizosphere. Funct. Ecol. 33:540-552.

Morella, N. M., Weng, F. C., Joubert, P. M., Metcalf, C. J. E., Lindow, S., and Koskella, B. 2020. Successive passaging of a plant-associated microbiome reveals robust habitat and host genotype-dependent selection. Proc. Natl. Acad. Sci. U.S.A. 117:1148-1159.

Moroenyane, I., Mendes, L., Tremblay, J., Tripathi, B., and Yergeau, É. 2021a. Plant compartments and developmental stages modulate the balance between niche-based and neutral processes in soybean microbiome. Microb. Ecol.

Moroenyane, I., Tremblay, J., and Yergeau, É. 2021b. Temporal and spatial interactions modulate the soybean microbiome. FEMS Microbiol. Ecol. 97: fiaa206.

Moscatiello, R., Baldan, B., and Navazio, L. 2013. Plant cell suspension cultures. Methods Mol. Biol. 953:77-93.
Nelson, E. B., Simoneau, P., Barret, M., Mitter, B., and Compant, S. 2018. Editorial special issue: The soil, the seed, the microbes and the plant. Plant Soil 422:1-5.

Oksanen J, Blanchet, F. G., Friendly, M., Kindt, R., Legendre, P., Minchin, P. R., O'Hara, R. B., Simpson, G. L., Solymos, P., Stevens, M. H. H., Szoecs, E., and Wagner, H. 2013. vegan: Community ecology package.

Özkurt, E., Hassani, M. A., Sesiz, U., Künzel, S., Dagan, T., Özkan, H., and Stukenbrock, E. H. 2020. Seed-Derived microbial colonization of wild emmer and domesticated bread wheat (Triticum dicoccoides and $T$. aestivum) seedlings shows pronounced differences in overall diversity and composition. MBio 11:e02637-20.

Pichinoty, F., Mandel, M., Greenway, B., and Garcia, J.-L. 1977. Isolation and properties of a denitrifying bacterium related to Pseudomonas lemoignei. Int. J. Syst. Evol. Microbiol. 27:346-348.

Poly, F., Monrozier, L. J., and Bally, R. 2001. Improvement in the RFLP procedure for studying the diversity of nifH genes in communities of nitrogen fixers in soil. Res. Microbiol. 152:95-103.

Quast, C., Pruesse, E., Yilmaz, P., Gerken, J., Schweer, T., Yarza, P., Peplies, J., and Glöckner, F. O. 2013. The SILVA ribosomal RNA gene database project: Improved data processing and web-based tools. Nucleic Acids Res. 41:D590-D596.

R Core Team. 2020. R: A Language and Environment for Statistical Computing. R Foundation for Statistical Computing, Vienna, Austria.

Ridout, M. E., Schroeder, K. L., Hunter, S. S., Styer, J., and Newcombe, G. 2019. Priority effects of wheat seed endophytes on a rhizosphere symbiosis. Symbiosis 78:19-31.

Robinson, R. J., Fraaije, B. A., Clark, I. M., Jackson, R. W., Hirsch, P. R., and Mauchline, T. H. 2016. Wheat seed embryo excision enables the creation of axenic seedlings and Koch's postulates testing of putative bacterial endophytes. Sci. Rep. 6:25581.

Rodríguez, C. E., Antonielli, L., Mitter, B., Trognitz, F., and Sessitsch, A. 2020. Heritability and functional importance of the Setaria viridis bacterial seed microbiome. Phytobiomes J. 4:40-52.

Sanz-Saez, A., Perez-Lopez, U., del-Canto, A., Ortiz-Barredo, A., Mena-Petite, A., Aranjuelo, I., Munoz-Rueda, A., and Lacuesta, M. 2019. Changes in environmental $\mathrm{CO}_{2}$ concentration can modify Rhizobium-soybean specificity and condition plant fitness and productivity. Environ. Exp. Bot. 162: 133-143.

Shade, A., Jacques, M. A., and Barrett, M. 2017. Ecological patterns of seed microbiome diversity, transmission, and assembly. Curr. Opin. Microbiol. 37:15-22.

Smith, M. E., Facelli, J. M., and Cavagnaro, T. R. 2018. Interactions between soil properties, soil microbes and plants in remnant-grassland and old-field areas: A reciprocal transplant approach. Plant Soil 433: 127-145.

Sommers, C. H. 2012. Microbial decontamination of food by irradiation. Pages 322-343 in: Microbial Decontamination in the Food Industry. A. Demirci and M. O. Ngadi, eds. Woodhead Publishing Series in Food Science, Technology and Nutrition. Woodhead Publishing Limited, Cambridge, U.K.

Sturz, A. V., Christie, B. R., and Nowak, J. 2000. Bacterial endophytes: Potential role in developing sustainable systems of crop production. Crit. Rev. Plant Sci. 19:1-30.

Stuy, J. H. 1960. Studies on the radiation inactivation of microorganisms. VI. $\mathrm{X}$-ray-induced breakdown of deoxyribonucleic acid in Haemophilus influenzae and in other bacteria. J. Bacteriol. 79:707-715.

Tannenbaum, I., Kaur, J., Mann, R., Sawbridge, T., Rodoni, B., and Spangenberg, G. 2020. Profiling the Lolium perenne microbiome: From seed to seed. Phytobiomes J. 4:281-289.

Toju, H., Peay, K. G., Yamamichi, M., Narisawa, K., Hiruma, K., Naito, K., Fukuda, S., Ushio, M., Nakaoka, S., Onoda, Y., Yoshida, K., Schlaeppi, K., Bai, Y., Sugiura, R., Ichihashi, Y., Minamisawa, K., and Kiers, E. T. 2018. Core microbiomes for sustainable agroecosystems. Nat. Plants 4 : 247-257.

Tourna, M., Freitag, T. E., Nicol, G. W., and Prosser, J. I. 2008. Growth, activity and temperature responses of ammonia-oxidizing archaea and bacteria in soil microcosms. Environ. Microbiol. 10:1357-1364.

Tremblay, J., and Yergeau, E. 2019. Systematic processing of ribosomal RNA gene amplicon sequencing data. Gigascience 8:giz146.

Truyens, S., Weyens, N., Cuypers, A., and Vangronsveld, J. 2015. Bacterial seed endophytes: Genera, vertical transmission and interaction with plants. Environ. Microbiol. Rep. 7:40-50. 
Wang, M., Eyre, A. W., Thon, M. R., Oh, Y., and Dean, R. A. 2020. Dynamic changes in the microbiome of rice during shoot and root growth derived from seeds. Front. Microbiol. 11:559728.

Wang, Q., Garrity, G. M., Tiedje, J. M., and Cole, J. R. 2007. Naive Bayesian classifier for rapid assignment of rRNA sequences into the new bacterial taxonomy. Appl. Environ. Microbiol. 73:5261-5267.

Xu, H., Sheng, R., Xing, X., Zhang, W., Hou, H., Liu, Y., Qin, H., Chen, C., and Wei, W. 2019. Characterization of fungal nirK-containing communities and $\mathrm{N}_{2} \mathrm{O}$ emission from fungal denitrification in arable soils. Front. Microbiol. 10:117.

Yang, J., Kloepper, J. W., and Ryu, C. M. 2009. Rhizosphere bacteria help plants tolerate abiotic stress. Trends Plant Sci. 14:1-4.
Yergeau, É., Quiza, L., and Tremblay, J. 2020. Microbial indicators are better predictors of wheat yield and quality than $\mathrm{N}$ fertilization. FEMS Microbiol. Ecol. 96:fiz205.

Zappala, S., Helliwell, J. R., Tracy, S. R., Mairhofer, S., Sturrock, C. J., Pridmore, T., Bennett, M., and Mooney, S. J. 2013. Effects of X-ray dose on rhizosphere studies using X-ray computed tomography. PLoS One 8:e67250.

Zhou, S.-Y.-D., Li, H., Giles, M., Neilson, R., Yang, X.-r., and Su, J.-q. 2021 a. Microbial flow within an air-phyllosphere-soil continuum. Front. Microbiol. 11:3325.

Zhou, S.-Y.-D., Zhang, Q., Neilson, R., Giles, M., Li, H., Yang, X.-R., Su, J.Q., and Zhu, Y.-G. 2021b. Vertical distribution of antibiotic resistance genes in an urban green facade. Environ. Int. 152:106502. 